Artigo Original

\title{
Tratamento Cirúrgico de Metástases Intracranianas
}

\author{
Surgery treatment of brain metastases
}

\author{
Franz J Onishi ${ }^{1}$, Julieta GSP Melo², Paulo MP Melo², Oreste P Lanzoni ${ }^{3}$, Flávio Settanni ${ }^{4}$, \\ Fernando AP Ferraz 5 .
}

\section{RESUMO}

Introdução: As metástases cerebrais formam o grupo de neoplasias mais freqüente do sistema nervoso central, e cerca de $40 \%$ dos pacientes com câncer desenvolvem metástases cerebrais. Pacientes com metástases únicas ou cujo quadro neurológico pode ser atribuído à lesão se beneficiam do tratamento cirúrgico. A evolução e o tratamento desta doença ainda permanecem controversos. Objetivos: Descrever as características clínicas e epidemiológicas dos pacientes com metástases cerebrais ressecadas no período de 2002 a 2003 no Hospital São Paulo. Métodos: Trabalho retrospectivo, descritivo, analítico, com coleta de dados baseada em revisão de prontuários de pacientes submetidos a ressecção cirúrgica de neoplasia secundária cerebral. Buscou-se a caracterização da população de estudo. Resultados: 30 pacientes foram avaliados (16 do sexo feminino). A média das idades dos pacientes foi de $52,9 \pm 11,7$ anos. As metástases de pulmão e mama compreenderam a maioria dos tumores operados. Houve desde então 12 óbitos registrados até a coleta de dados, com média de sobrevida pós-operatória de 72,92 dias, sendo o pior prognóstico relacionado às metástases de melanoma. Conclusão: As metástases cerebrais constituem ainda um grupo de doenças com prognóstico reservado, acometendo adultos economicamente ativos. Os pacientes com metástases de tumores de mama apresentaram tendência a maior sobrevida. A comparação de dados de diferentes centros é essencial para estabelecer a melhor conduta no tratamento destes casos.

Unitermos: Tumores SNC, Metástases cerebrais, Neoplasias secundárias.

Citação: Onishi FJ, Melo JGSP, Melo PMP, Lanzoni OP, Settanni F, Ferraz FAP. Tratamento Cirúrgico de Metástases Intracranianas. Rev Neurociencias 2005; 13(1):011-016.

\section{SUMMARY}

Introduction: Cerebral metastases are the most frequent group of central nervous system tumors, and up to $40 \%$ of cancer patient's develop a brain metastasis. Single metastasis or neurological problems related to the metastasis injury can have benefits from the surgical treatment, besides patient's evolution and even the treatment remains controversial. Objectives: To describe clinical and epidemiological features of patients with cerebral metastases resected from 2002 to 2003. Methods: This is a retrospective, descriptive and analytic study where data were collected from patients' charts. All neurosurgically treated patients were included. Results: Thirty patients (16 female) were studied

Trabalho realizado: Disciplina de Neurocirurgia - Universidade Federal de São Paulo - UNIFESP.

1 - Médico Residente da Disciplina de Neurocirurgia - UNIFESP

2 - Pós-graduandos da Disciplina de Neurocirurgia - UNIFESP

3 - Preceptor de Residentes da Disciplina de Neurocirurgia - UNIFESP

4 - Professor Livre Docente da Disciplina de Neurocirurgia - UNIFESP

5 - Professor Adjunto da Disciplina de Neurocirurgia - UNIFESP

Endereço para correspondência: Dr. Franz J Onishi

Rua Napoleão de Barros, $715,6^{\circ}$ andar.

São Paulo - SP - Brasil - CEP 04024-002

E-mail: franzonishi@gmail.com

Trabalho recebido em 26/01/05. Aprovado em 02/05/05 
(mean age $52.9 \pm 11.7$ years). Lung and breast metastasis predominated. Twelve patients have died since we start registering data (mean survival time: 72.92 days), and those related to melanoma metastasis had the worst evolution. Conclusion: Brain metastases are a still reserved regarding prognosis and unfortunately affects economically active people like our series. Breast cancer metastases had a better prognosis in our sample but we need to compare our data to other Brazilian centers to discuss and establish the appropriate treatment to those patients.

\section{Keywords: CNS tumors, Cerebral metastases, Secondary neoplasia.}

Citation: Onishi FJ, Melo JGSP, Melo PMP, Lanzoni OP, Settanni F, Ferraz FAP. Surgery treatment of brain metastases. Rev Neurociencias 2005; 13(1):011-016.

\section{INTRODUÇÃO}

As neoplasias secundárias são as lesões tumorais mais freqüentes no sistema nervoso central, representando uma importante causa de morbidade e mortalidade por câncer. Com o desenvolvimento de métodos diagnósticos mais sensíveis e com a maior difusão e acesso a eles, as metástases cerebrais vêm sendo diagnosticadas com maior freqüência. De todos os pacientes com diagnóstico de neoplasia maligna, 20 a 40\% desenvolvem metástases para o cérebro ${ }^{1-3}$. Câncer de pulmão, mama e o melanoma são as neoplasias sistêmicas mais relacionadas à doença. Em aproximadamente $20 \%$ dos casos o tumor primário é oculto, sendo esta a primeira manifestação da doença sistêmica ${ }^{4}$.

Embora o parênquima cerebral seja a região mais acometida, encontram-se lesões secundárias também na calota craniana e na dura-máter. Em pacientes com câncer, o encéfalo constitui um dos sítios de acometimento mais debilitantes e que causa grande morbidade, pelo potencial de déficits motores e cognitivos ${ }^{5-7}$.

Aproximadamente metade dos pacientes apresenta cefaléia como o primeiro sintoma. Déficit motor, alteração do estado mental e crises convulsivas também estão presentes, em ordem decrescente de freqüência. O diagnóstico é estabelecido por métodos de neuroimagem e o prognóstico depende do estágio da doença sistêmica ${ }^{5}$.

Por muitos anos, o tratamento padrão das metástases cerebrais foi a radioterapia de encéfalo total (RT), com doses variáveis e fracionadas de radiação. Mais recentemente, a radiocirurgia estereotática e a ressecção microcirúrgica vêm se mostrando mais efetivas para o tratamento de tais doenças, com sobrevida mais longa ${ }^{4,8-12}$.

O tratamento multidisciplinar pode determinar aumento na sobrevida. Foram determinados na literatu- ra fatores de bom prognóstico pós-tratamento: doença intracraniana limitada, bom estado clínico, doença primária controlada, faixa etária jovem e menor agressividade do tipo histológico metastático ${ }^{12-15}$.

Atualmente, os estudos apontam que o melhor tratamento para metástase cerebral única é a ressecção cirúrgica, quando possível, seguida de radioterapia. Esta conduta aumenta a sobrevida e principalmente o tempo de independência funcional do paciente. O tratamento cirúrgico também está indicado em lesões cujo tumor primário é desconhecido e em lesões que produzam hipertensão intracraniana $3-4,12,16-17$.

Nas metástases múltiplas, a conduta inicial é a ressecção cirúrgica da lesão sintomática, seguida de radiocirurgia nas demais lesões, ou radioterapia convencional. Para cada caso deve-se, conhecendo a história natural da neoplasia, determinar o custo-benefício deste tratamento 4 .

A radiocirurgia é eficaz no controle local das lesões, mas parece não afetar de forma significativa a sobrevida de pacientes com múltiplas metástases, já que geralmente o controle intracraniano é eficaz, mas a doença extra-craniana progride. Em pacientes selecionados, que possuem boas condições neurológicas, a radiocirurgia diminui a progressão das metástases e melhora a sobrevida cognitiva do paciente ${ }^{18-21}$.

A dose do tratamento com radioterapia estereotáxica ou radiocirurgia é inversamente proporcional ao volume de tratamento. Segundo este princípio, metástases pequenas podem tolerar doses crescentes de radiação ${ }^{20}$.

Em uma grande série, a sobrevida média dos pacientes submetidos à radiocirurgia foi de 9 meses. Lesões decorrentes de melanoma ${ }^{19}$ e lesões múltiplas determinaram uma sobrevida menor. 0 controle da doença local em 1 ano após a 
radiocirurgia foi de 75\%. A associação de radiocirurgia e radioterapia de encéfalo total não demonstrou melhor evolução quando comparada ao tratamento radiocirúrgico isolado ${ }^{18}$. Outro estudo demonstrou controle de doença em 1 ano de $69 \%$ e $46 \%$ no segundo ano. Quando avaliados apenas tumores com até $1 \mathrm{~cm}\left(0,5 \mathrm{~cm}^{3}\right)$ esta taxa sobe para 86 e $78 \%$ respectivamente, contra $56 \%$ e $24 \%$ quando maiores que este tamanho ${ }^{20}$.

Quimioterápicos têm sido empregados no tratamento das metástases cerebrais de acordo com a indicação clínica da doença primária, alguns deles em fases experimentais com resultados ainda inconclusivos $22-23$.

\section{OBJETIVOS}

Caracterizar clinicamente os pacientes submetidos à ressecção cirúrgica de metástases intracranianas, analisar fatores de sobrevida, estudo de sítio primário mais comum, descrição de localização tumoral, análise individualizada por gênero e idade média ao diagnóstico.

\section{MÉTODOS}

Realizada análise retrospectiva de todos os pacientes adultos com diagnóstico histológico de metástase cerebral, operados no Hospital São Paulo - Universidade Federal de São Paulo e acompanhados no ambulatório de Neurocirurgia no período compreendido entre janeiro de 2002 e dezembro de 2003. Realizada avaliação dos dados clínicos dos pacientes baseada em coleta de dados nos respectivos prontuários médicos. Elaborado um estudo retrospectivo descritivo e analítico utilizando o programa SPSS 12.0 para tratamento estatístico dos dados coletados.

Foram excluídos pacientes com doença primária fora de controle, pacientes com múltiplas metástases e pacientes sem condições clínicas pré-operatórias para a indicação do tratamento cirúrgico.

Os dados foram analisados através dos testes de Kruskal-Wallis, Rank test, teste de correlação de Pearson e teste t de Student.

\section{RESULTADOS}

Foram incluídos 30 pacientes consecutivos, com idade média de 52,9 $( \pm 11,7)$ anos, portadores de metástases intracranianas submetidos à ressecção cirúrgica, com confirmação histológica de neoplasia secundária. Quatorze pacientes (46,7\%) eram do sexo masculino e dezesseis $(53,3 \%)$ do sexo feminino. O tempo de seguimento médio dos pacientes foi de 548 dias.

Das neoplasias, isolamos os seguintes tipos histológicos em ordem de freqüência: câncer de pulmão e mama correspondendo cada um a 26,7\% ( $n=8$, em cada grupo), melanoma em $20 \%(n=6)$ e outros tipos histológicos $26,7 \%(n=8)$. Entre estes encontramos carcinoma de células claras renais, carcinomas indiferenciados de sítio primário indefinido, carcinoma folicular de tireóide, adenocarcinoma de origem indeterminada. Metástases cerebrais foram a manifestação inicial de neoplasias malignas sistêmicas em 4 pacientes $(13,3 \%)$.

O quadro clínico inicial foi constituído por cefaléia em $60 \%$ dos casos, não obedecendo um caráter típico, crises convulsivas (16,7\%), déficit focal (16,7\%) e rebaixamento do nível de consciência $(6,7 \%)$. No momento da cirurgia, $46,7 \%$ dos pacientes já apresentavam déficit focal.

O lobo frontal foi o local mais acometido $(36,7 \%)$, seguido pelo parietal (20\%) e cerebelo $(16,7 \%)$.

A radioterapia pós-operatória foi administrada a $90 \%$ dos pacientes. A taxa de mortalidade geral após 18 meses de acompanhamento foi de 40\%, com média de sobrevida de 172,9 \pm 88 dias. A média de idade foi de 53,3 anos entre os pacientes do grupo com evolução desfavorável, semelhante à média etária global da população selecionada para este estudo.

Não houve diferença estatisticamente significante em relação à idade, quando analisamos a proporção de metástases cerebrais no sexo masculino e feminino ((F:52,4 M:53,4; $p=0,8)$.A sobrevida pós-cirúrgica variou, mas não diferiu estatisticamente para metástases de tumores pulmonares, melanoma e da mama $(p=0,09)$. Houve uma discreta tendência de pacientes mais idosos apresentarem menor sobrevida $(r=-0,215 ; p=0,502)$.

\section{DISCUSSÃO}

As metástases cerebrais são as neoplasias mais prevalentes do sistema nervoso central, acometem até $40 \%$ dos pacientes com câncer. Grande parte 
dos estudos apresenta uma prevalência masculina maior, embora sem relevância estatística. Nesta série apresentamos uma discreta predominância no sexo masculino, provavelmente pelo grande número porcentual de neoplasia de mama quando comparado a outras séries (Figura 1 ).

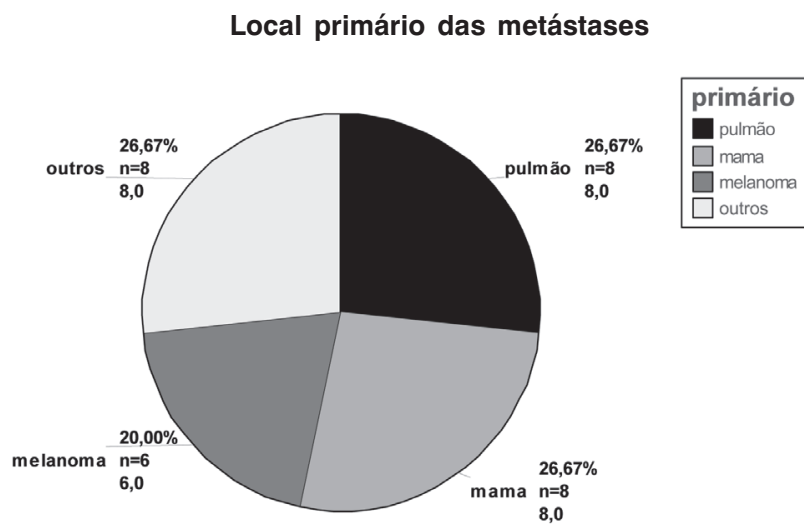

Figura 1. Amostragem dos pacientes submetidos a ressecção cirúrgica de lesões secundárias cerebrais.

Apesar de não ser estatisticamente significante, no sexo feminino o diagnóstico de metástase cerebral se deu em uma faixa etária menor que no sexo masculino. Esta diferença também pode ser explicada pela presença das metástases secundárias a tumor de mama, diagnosticadas em uma faixa etária menor que as secundárias a tumores pulmonares ou melanomas (48, 56,7 e 57,6 anos, respectivamente) (Figura 2).

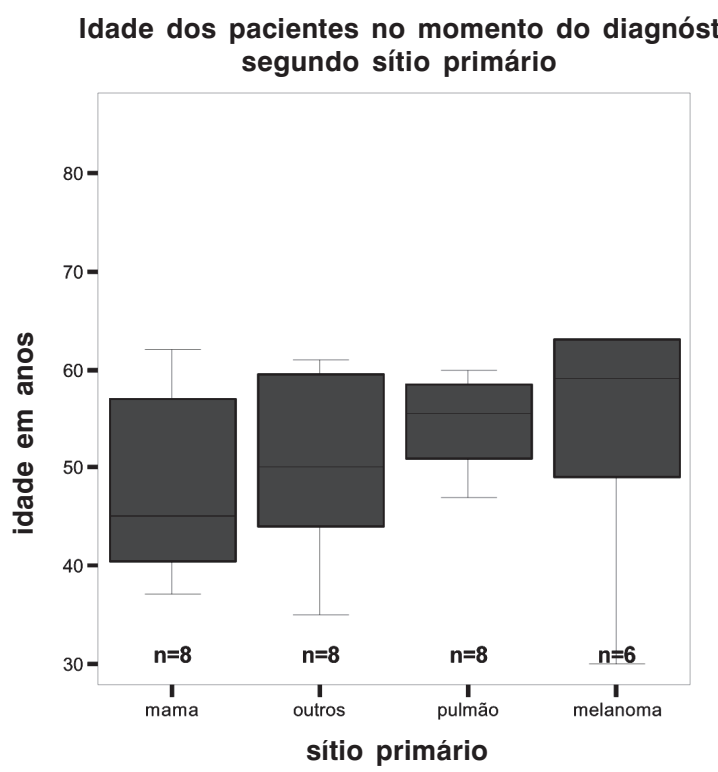

Figura 2. Idade dos pacientes do estudo segundo o sítio primário.
O Grupo de Tumores da Disciplina de Neurocirur-gia da Escola Paulista de Medicina adotou os critérios clássicos estabelecidos na literatura para a indicação do tratamento cirúrgico das metástases. Desta forma, os pacientes com metástases únicas ao diagnóstico e os pacientes com lesões sintomáticas foram submetidos à ressecção destas lesões e encaminhados para tratamento adjuvante com radioterapia e quimioterapia. Por este estudo tratar de um grupo selecionado de pacientes cirúrgicos, o quadro clínico descrito não é o mesmo encontrado no grupo geral dos pacientes com metástases cerebrais. Neste grupo o quadro clínico inicial de síndrome de hipertensão intracraniana abrangeu $66 \%$ dos pacientes com cefaléia e sonolência, 46,7\% dos pacientes apresentavam déficit neurológico focal no momento da cirurgia e apenas 16,7\% apresentavam apenas crise convulsiva de início tardio.

Assim como na literatura, a maior parte das lesões secundárias cerebrais neste grupo foram supratentorias $(73,3 \%)$ e $16,7 \%$ cerebelares. Não foi encontrada relação estatística entre a localização da lesão e o tipo histológico.

Nesta série de casos obtivemos índices de mortalidade em 18 meses semelhantes aos da literatura (40\%). A média de sobrevida também se sobrepõe à dos estudos revistos.

Até a compilação dos dados, 12 pacientes haviam falecido, $50 \%$ deles com diagnóstico de metástases de tumores pulmonares, com sobrevida média pós-cirúrgica de 53 dias. Apenas uma paciente portadora de metástase de adenocarcinoma mamário havia falecido, com sobrevida de 311 dias. Já os melanomas, acometendo pacientes com faixa etária mais elevada, haviam gerado duas mortes, com sobrevida média de 6,5 dias. Apesar da grande variância, não se observa significância estatística entre a sobrevida dos 3 grupos (Kruskal-Wallis on Ranks, $p=0,09)$. O coeficiente de letalidade observado foi maior, portanto, em pacientes com neoplasia pulmonar. Entre os pacientes falecidos, os pacientes com melanoma tiveram menor sobrevida pós-cirúrgica (Figura 3).

Observamos uma correlação negativa, no entanto sem significância estatística, entre a idade do paciente operado e a sobrevida pós-cirúrgica, o que significa dizer que os pacientes mais ido- 


\section{Tempo de vida pós-operatória, segundo sítio primário}

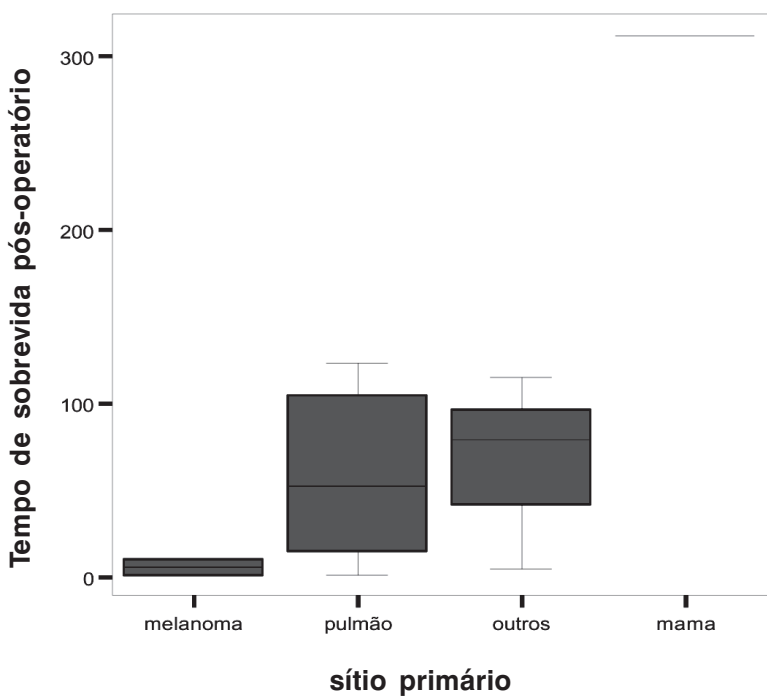

Figura 3. Tempo de sobrevida pós-operatória dos pacientes em dias (n de óbitos até a compilação dos dados $=12$ ).

sos tenderam a ter menor sobrevida pós-cirúrgica (Pearson $r=-0,215 p=0,502$ ) (Figura 4).

\section{CONCLUSÃO}

Pacientes com metástases cerebrais únicas ou sintomáticas, quando submetidos a tratamento cirúrgico em tempo adequado e terapia adjuvante se beneficiam com aumento da sobrevida. Além disto, há melhora na qualidade de vida graças à resolução da hipertensão intracraniana ou dos déficits

\section{Correlação entre idade dos pacientes e o tempo de sobrevida}

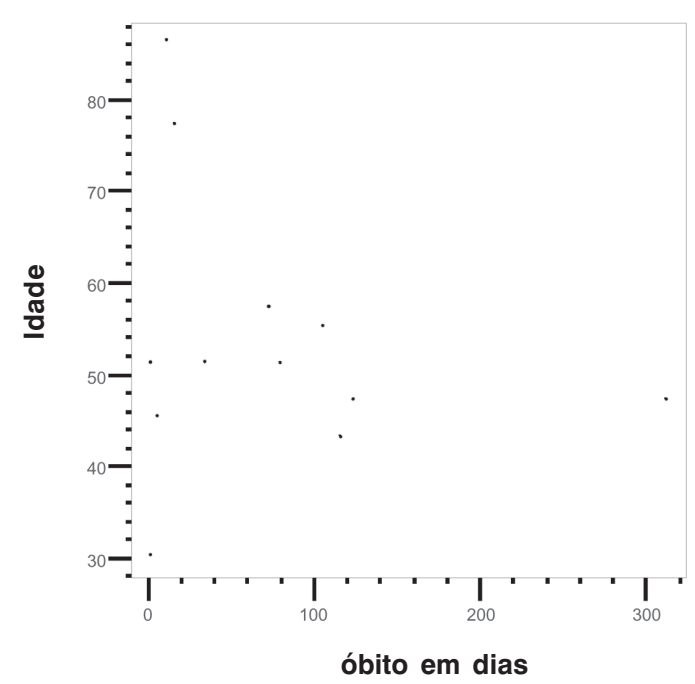

Figura 4. Correlação entre a idade dos pacientes e o tempo de sobrevida pós-cirúrgico.

focais. O tratamento cirúrgico deve ser proposto aos pacientes com metástase cerebral quando houver lesão única, em local acessível, e controle sistêmico da neoplasia primária; em lesões múltiplas, devendo ser ressecada a sintomática e as demais que possam ser removidas pela mesma via de acesso. O tratamento adjuvante é importante para controle local da doença. Neste estudo, pacientes com metástase de neoplasia mamária apresentaram evolução mais favorável, enquanto pacientes com lesões secundárias a melanoma ou tumor pulmonar apresentaram sobrevida menor.

\section{REFERÊNCIAS BIBLIOGRÁFICAS}

1. Black PM, Johnson MD. Surgical resection for patients with solid brain metastases: current status. J Neurooncol 2004 69(1-3): 119-24

2. Nguyen T, Deangelis LM. Treatment of brain metastases. J Support Oncol 2004; 2(5):405-10 (discussion 411-6).

3. Bradley KA, Mehta MP. Management of brain metastases. Semin Oncol 2004; 31(5):693-701.

4. Kondziolka D, Patel A, Lunsford LD, Flickinger JC. Decision making for patients with multiple brain metastases: radiosurgery, radiotherapy, or resection? Neurosur Focus 2000; 9:1-6.

5. Barnholtz-Sloan JS, Sloan AE, Davis FG, Vigneau FD, Lai $P$, Sawaya RE. Incidence Proportions of Brain Metastases In-Patients Diagnosed (1973 to 2001) in the Metropolitan Detroit Cancer Surveillance System. J clin oncol 2004; 22(14): 2865-2872.
6. Takeshima H, Kuratsu J, Nishi T, Ushio Y. Prognostic Factors in Patients Who Survived More Than 10 Years after Undergoing Surgery forMetastatic Brain Tumors: Report of 5 cases and review of the literature. Surg Neurol 2002; 58:118-23.

7. Chang EL, Hassenbusch SJ 3rd, Shiu AS, et al. The role of tumor size in the radiosurgical management of patients with ambiguous brain metastases. Neurosur 2003; 53(2): 272-80.

8. Ulm AJ, Friedman WA, Bova FJ, Bradshaw P, Amdur RJ, Mendenhall WM. Linear Accelerator Radiosurgery in the Treatment of Brain Metastases. Neurosur 2004; 55(5):1076-1085.

9. Okunieff P, Schell MC, Ruo R, Hale ER, O'Dell WG, Pilcher W. Long-term management of patients with multiple brain metastases after shaped beam radiosurgery. Case report and review of the literature. J Neurosurg 2004; 101 Suppl 3:406-12. 
10. Warnick RE, Darakchiev BJ, Breneman JC. Stereotactic radiosurgery for patients with solid brain metastases: current status. J Neurooncol 2004; 69(1-3):125-37.

11. Schöggl A, Kitz K, Reddy M, Wolfsberger St, Schneider B, Dieckmann K, Ungersböck K. Defining the Role of Stereotactic Radiosurgery Versus Microsurgery in the Treatment of Single Brain Metastases. Acta Neurochir (Wien) 2000; 142:621-626.

12. Sheehan J, Niranjan A, Flickinger JC, Kondziolka D, Lunsford LD. The Expanding Role of Neurosurgeons in the Management of Brain Metastases. Surg Neurol 2004; 62:32-41.

13. Kimura T, Sako K, Tohyama $Y$, et al. Diagnosis and treatment of progressive space-occupying radiation necrosis following stereotactic radiosurgery for brain metastasis: Value of proton magnetic resonance spectroscopy. Acta Neurochir 2003, 145:557-564.

14. Pierallini A, Caramia F, Piattella MC, et al. Metastasis Along the Stereotactic Biopsy Trajectory in Glioblastoma Multiforme. Acta Neurochir (Wien) 1999; 141:1011-1012.

15. Bajard A, Westeel V, Dubiez A, et al. Multivariate analysis of factors predictive of brain metastases in localised non-small cell lung carcinoma. Lung Cancer 2004; 45:317-323.

16. Rock JP, Haines S, Recht $L$, et al. Practice parameters for the management of single brain Metastasis. Neurosur Focus 2000; $9: 1-9$
17. Mystakidou K, Boviatsis EJ, Kouyialis AT, et al. Silent radiological imaging time in patients with brain metastasis. Clin Neurol Neurosur 2004; 106: 300-304.

18. Shehata MK, Reid BYB, Patchell RA, et al. Stereotatic radiosurgery of 468 brain metastasis $<2 \mathrm{~cm}$ : Implications for SRS dose and whole brain radiation therapy. Int $\mathrm{J}$ Rad Oncol Biol Phys 2004; 59(1): 87-93.

19. Selek U, Chang EL, Hassenbusch III SJ, et al. Stereotatic radiosurgical treatment in 103 patientes for 153 cerebral melanoma metastases. Int. J. Rad Oncol Biol Phys 2004; 59(4): 1097-1106.

20. Datta R, Jawahar A, Ampil FL, Shi R, Nanda A, D'Agostino H. Survival in Relation to Radiotherapeutic Modality for Brain Metastasis. Whole Brain Irradiation vs. Gamma Knife Radiosurgery. Am J Clin Oncol 2004; 27(4): 420-424.

21. Kim DG, Kim CY, Paek SH, et al. Whole-Body [18F] FDG PET in the Management of Metastatic Brain Tumours. Acta Neurochir (Wien) 1998; 140:665-674.

22. Poon AN, Ho SS, Yeo W, Mok TS. Brain metastasis responding to gefitinib alone. Oncol 2004; 67(2): 174-8.

23. Chiocca EA, Broaddus WC, Gillies GT, Visted T, Lamfers MLM. Neurosurgical delivery of chemotherapeutics, targeted toxins, genetic and viral therapies in neuro-oncology. J Neuro-Oncol 2004; 69:101-117. 